\title{
Enhancement of Electrocatalytic Oxygen Evolution by Chiral Molecular Functionalization of Hybrid 2D Electrodes
}

Yunchang Liang, ${ }^{1,2,{ }^{*}}$ Karla Banjac, ${ }^{1,2}$ Kévin Martin, ${ }^{3}$ Nicolas Zigon, ${ }^{3}$ Seunghwa Lee, ${ }^{4}$ Nicolas Vanthuyne, ${ }^{5}$ Felipe Andrés Garcés, ${ }^{6}$ José R. Galan-Mascaros,${ }^{6,7}$ Xile Hu, ${ }^{4}$ Narcis Avarvari, ${ }^{3,}$ Magalí Lingenfelder ${ }^{1,2^{*}}$

${ }^{1}$ Max Planck-EPFL Laboratory for Molecular Nanoscience and Technology, École Polytechnique Fédérale de Lausanne (EPFL), 1015 Lausanne, Switzerland

${ }^{2}$ Institut of Physics (IPHYS), Ecole Polytechnique Fédérale de Lausanne (EPFL), 1015 Lausanne, Switzerland

${ }^{3}$ Univ Angers, CNRS, MOLTECH-Anjou, SFR MATRIX, F-49000 Angers, France

${ }^{4}$ Laboratory of Inorganic Synthesis and Catalysis, Institute of Chemical Sciences and Engineering, École Polytechnique Fédérale de Lausanne (EPFL), 1015 Lausanne, Switzerland

${ }^{5}$ Aix Marseille Université, CNRS, Centrale Marseille, iSm2, Marseille, France

${ }^{6}$ Institute of Chemical Research of Catalonia (ICIQ), The Barcelona Institute of Science and Technology (BIST), Av. Països Catalans 16, E-43007 Tarragona, Spain

${ }^{7}$ Catalan Institution for Research and Advanced Studies (ICREA), Passeig Lluis Companys, 23, Barcelona 08010, Spain

*e-mail: yunchang.liang@epfl.ch; narcis.avarvari@univ-angers.fr; magali.lingenfelder@epfl.ch 


\begin{abstract}
A sustainable future requires highly efficient energy conversion and storage processes, where electrocatalysis plays a crucial role. The activity of an electrocatalyst is governed by the binding energy towards the reaction intermediates, while the scaling relationships prevent the improvement of a catalytic system over its volcano-plot limits. To overcome these limitations, unconventional methods that are not fully determined by the surface binding energy can be helpful. Here, we use organic chiral molecules, i.e., hetero-helicenes, to boost the oxygen evolution reaction (OER) by ca. $131.5 \%$ (at the potential of $1.65 \mathrm{~V}$ vs. $\mathrm{RHE}$ ) at state-of-the-art 2D catalysts via a spin-polarization mechanism. Our results show that chiral molecule-functionalization is able to increase the OER activity of catalysts beyond the volcano limits. A guideline for optimizing the catalytic activity via chiral molecular functionalization of hybrid 2D electrodes is given.
\end{abstract}




\section{Main}

Efficient electrocatalysis, especially electrocatalytic water-splitting, is imperative in future sustainable energy systems and carbon neutrality processes ${ }^{1,2}$. However, the complex reaction pathways and sluggish kinetics of the oxygen evolution reaction (OER) impede the application of electrocatalytic water-splitting ${ }^{3}$. The Sabatier principle states that an ideal catalyst surface should bind to the reaction intermediates with optimal strength (neither too weak nor too strong $)^{4}$. Moreover, the scaling relationships correlate the binding energy of the variables of one adsorbate species to a catalyst surface; consequently, a volcano-type plot confines the activity of the catalysts and guides the development of new catalytic surfaces $^{5,6,7}$. Therefore, conventional strategies to enhance the catalytic activity are mostly based on adjusting the binding energy. Unconventional approaches including magnetic control can improve the electrocatalysis efficiency beyond the volcano limitations and are receiving increasing attention $8,9,10$.

Electron transfer takes place during electrocatalysis processes. One of the inherent properties of electrons is the electron spin. Recently, it was reported that electron transport through a chiral molecule induces a spin polarization without external magnetic fields, i.e., after passing the molecule an imbalance between up and down spin is created ${ }^{11,12}$. This socalled chirality induced spin selectivity (CISS) effect was suggested applicable in spindependent electron transfer processes at liquid/solid interfaces, for instance, the electrocatalytic OER ${ }^{8,13}$. It was suggested that the CISS process affects the spin of the electrons at the catalyst surface, consequently influencing the reaction pathway and enhancing the oxygen evolution. Therefore, it provides a unique opportunity to improve the activity and selectivity of the OER beyond the volcano plot-related restrictions.

However, experimentally decoupling the various coexisting effects contributing to OER is essential to assess the effect of chiral molecular functionalization. In particular, the high dissolution rate of catalyst materials (e.g., Fe) under OER conditions ${ }^{14}$, the weak bonding of thiol groups on metal oxides ${ }^{15,16}$, the influence of organic molecules and their different 
structural conformers on the electrochemically active surface area (ECSA) and the coordination environment of the active surface sites ${ }^{17}$ need to be considered. Therefore, well-defined systems are essential to decouple the effects of chiral molecular functionalization on the OER activity. Here, we use hybrid electrodes composed of a monolayer or sub-monolayer of rigid chiral/achiral molecules and 2D electrocatalysts in a sandwich configuration.

$2 \mathrm{D} \mathrm{Ni}\left(\mathrm{NiO}_{x}\right)$ and $\mathrm{NiFe}$ oxo-hydroxides are known as state-of-the-art electrocatalysts in alkaline media ${ }^{18}$. Carbohelicenes contain ortho-condensed aromatic rings with helical structures that possess axial chirality, and 2D self-assembly of helicene molecules on metals bestow chiral properties to the surface ${ }^{19}$. Their high racemization barriers ${ }^{20}$ preserve their enantiopurity at the liquid/solid interface during the OER. The rigid structure of the helicenes also limits the number of molecular conformers at the electrode surface $21,22,23,24$. However, carbohelicenes interact weakly with metallic substrates (for instance, $\mathrm{Cu}, \mathrm{Ag}$, and $\mathrm{Au}$ ) via van der Waals forces, resulting in high mobility of the molecules at room temperature ${ }^{19,25}$. Attachment of additional functional groups on helicenes is thus needed to increase the stability of such a hybrid electrode by strengthening the bonding to the metal surface ${ }^{26}$. Here, using chiral fused thiadiazole-helicene molecules, we report on the role of spin polarization by chiral molecular functionalization on the OER activity of metallic electrodes and the optimization of the chiral molecule-catalyst configuration for optimal activity.

\section{Results and discussion}

\section{Effect of the molecular functionalization on the OER activity}

In the first set of experiments, enantiopure $(M)$ - and $(P)$-thiadiazole-[7]helicenes, previously reported by some of $\mathrm{us}^{27}$ in the frame of our general interest in chiral molecular materials ${ }^{28}$, have been used for the chiral molecular functionalization of metal electrodes. 
Fig. 1 shows the effect of the adsorbed thiadiazole-[7]helicene enantiomers on the OER activity of two types of electrodes, namely $\mathrm{Au}(111)$ with monolayer $\mathrm{NiO}_{x}$ islands and bare $\mathrm{Au}(111)$, in $\mathrm{O}_{2}$-saturated $0.1 \mathrm{M}$ aqueous $\mathrm{KOH}$ solution. Farhat et al. ${ }^{29}$ reported that the OER activity of ultrathin $\mathrm{NiO}_{x}$ and $\mathrm{NiFeO}_{x}$ films under reaction conditions decreases over time in Fe-free $\mathrm{KOH}$ but stays more constant in unpurified (i.e., containing trace $\mathrm{Fe}$ impurities) $\mathrm{KOH}$ after Fe incorporation. Therefore, an unpurified $\mathrm{KOH}$ solution has been used to ensure all activity measurements are reproducible.

A clear enhancement in the OER current can be seen after the helicene molecules were deposited on $\mathrm{NiO}_{x}$ samples, as shown in Fig. 1 a and $\mathbf{b}$. Deposition of $(P)$ - and $(M)$ thiadiazole-[7]helicene enhances the OER current at $1.65 \mathrm{~V}$ vs. RHE approximately $85 \%$ and $74 \%$, respectively. Electrochemical impedance spectroscopy (EIS) measurements were conducted before and after the molecule deposition to determine if the current enhancement could be just due to an increment in the ECSA during the molecule deposition process. The ECSA (proportional to the active surface) was obtained by determining the specific adsorption capacitance around the "onset" potential of the $\mathrm{OER}^{30}$ (Supplementary Figure 1 a). The OER currents normalized by the ECSA values still uphold the enhancement effect, i.e., ca. $61 \%$ increase in the current density at $1.65 \mathrm{~V}$ vs. RHE and ca. $33.4 \mathrm{mV}$ reduction in the overpotential at the current density of $10 \mathrm{~mA} \mathrm{~cm}^{-2}$ in the case of $(P)$-thiadiazole[7]helicene (Supplementary Figure 1 b). It is likely that the increase in the specific adsorption capacitance in Supplementary Figure 1 a was mainly induced by the adsorbed helicene molecules ${ }^{31,32}$, and not by the increment of the ECSA. Nevertheless, the normalized current reinforces the conclusion that the thiadiazole-[7]helicene enantiomers are able to enhance the OER at $\mathrm{NiO}_{x}$ islands on Au surfaces.

Although the effectiveness of the helicene molecules on OER enhancement at $\mathrm{NiO}_{x}$ is validated, it is likely that molecules were not directly adsorbed on the $\mathrm{NiO}_{\mathrm{x}}$ islands as no metallic Ni sites were accessible during the measurements. Direct thiadiazole bonding on metal oxide surfaces is difficult under the current conditions ${ }^{33}$. Therefore, the molecules were 
mostly adsorbed on the Au substrate. To test the effect of direct bonding between the helicene molecule and the catalyst surface on the OER, bare $\mathrm{Au}(111)$ electrodes were used for analogous OER experiments (Fig. 1 c). Although such chirally functionalized $\mathrm{Au}(111)$ has lower OER activity compared to $\mathrm{NiO}_{x}$, we anticipate that the activity measurements on $\mathrm{Au}(111)$ help to elucidate the effect of direct adsorption of helicene molecules at OER active sites. In contrast to the results from $\mathrm{NiO}_{x}$ samples, the presence of $(P)$-thiadiazole[7]helicene molecules reduced the OER at the $\mathrm{Au}(111)$ surface. This difference can be ascribed to the different catalyst-helicene molecule configurations. At the $\mathrm{NiO}_{x}$ decorated $\mathrm{Au}$ substrates, the molecules were mostly adsorbed on the Au surfaces, and the active centers at $\mathrm{NiO}_{x}$ islands were not directly affected. At bare Au samples, the OER was taking place at the Au surface, which was partially blocked by the molecules after deposition. The exposed surface area of the Au samples was determined by the stripping of $\mathrm{Pb}$ atoms deposited by the underpotential deposition (UPD) method $^{34}$ (see Supplementary Figure $\mathbf{2} \mathbf{a}$ and $\mathbf{b}$ for the $\mathrm{Pb}$ stripping results and the normalized current density). The results show that a submonolayer of helicene molecules was on the sample surface after the deposition. The decrease in the OER current is not proportional to the decrease in the surface area of Au. The helicene molecules even reduced the specific current density. As shown in Supplementary Figure 2 a, peak 1, which can be assigned to low-coordination and step sites $^{34,35}$, decreased and shifted towards more negative potentials. Zwaschka et al. ${ }^{36}$ found that OER activity on a polycrystalline Au surface is dominated by $<1 \%$ of the surface consisting of defects. Therefore, the molecules were preferentially adsorbed on surface defects, or the molecule-metal bond at defect sites was more stable during the reactions. 

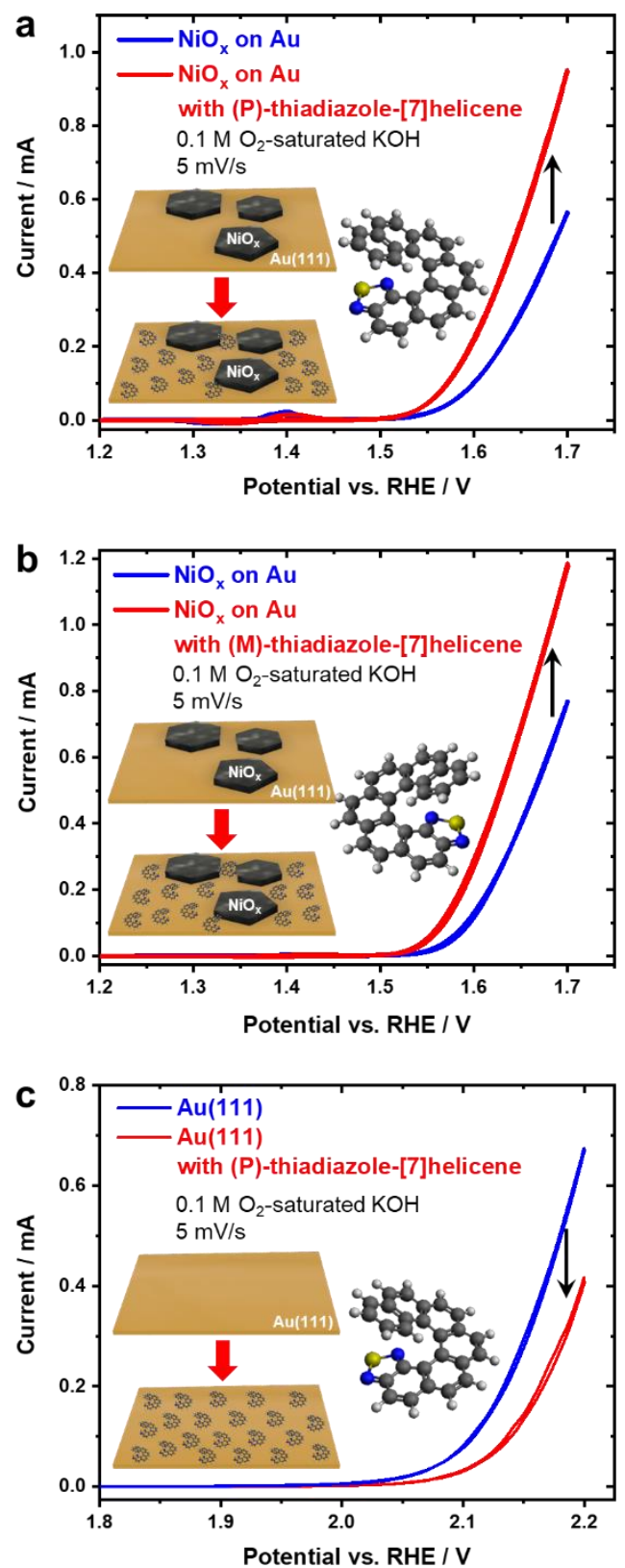

Fig. 1 | Helicene enantiomer functionalization effect on the OER activity. OER activity of a $(P)$-thiadiazole-[7]helicene and $\mathbf{b}(M)$-thiadiazole-[7]helicene embedded $\mathrm{NiO}_{x}$ electrodes in $0.1 \mathrm{M} \mathrm{O}_{2}$-saturated $\mathrm{KOH}$. 


\section{Molecular functionalization vs. Fe doping effect on OER}

Trotochaud et al. studied the effect of Fe impurities in the electrolyte and Fe doping in the catalysts on the OER activity, showing that Fe doping is efficient to improve the OER activity $^{37}$. However, this improvement still adheres to the volcano-plot limits ${ }^{38,39}$. In this work, Fe impurities from the electrolyte had been incorporated in the $\mathrm{NiO}_{x}$ samples during activity measurements before chiral molecular functionalization. A comparison of the activity enhancement caused by the $(P)$-thiadiazole-[7]helicene and the Fe doping in Ni-based catalysts is shown in Fig. 2. The $\mathrm{Ni}_{9} \mathrm{FeO}_{x}$ catalyst was deposited on $\mathrm{Ni}$ foils using a combustion method introduced elsewhere ${ }^{40}$. The ECSA was obtained from the determination of the specific adsorption capacitance ${ }^{30}$. The helicene molecules further increased the OER activity of highly active Ni-based catalysts modified by Fe doping. The enhancement was higher on $\mathrm{NiO}_{x}$ than on $\mathrm{Ni}_{9} \mathrm{FeO}_{x}$, e.g., ca. $93.8 \%$ on $\mathrm{NiO}_{x}$ and ca. $25.9 \%$ on $\mathrm{Ni}_{9} \mathrm{FeO}_{x}$ at the potential of $1.6 \mathrm{~V}$ vs. RHE, respectively. It is presumably caused by the different amounts of helicene molecules that can be embedded into different electrodes. On the $\mathrm{NiO}_{x}$ electrodes, helicene molecules were stably bonded to the Au substrates. However, the $\mathrm{Ni}_{9} \mathrm{FeO}_{x}$ electrode surface only consists of metal oxides, which do not form strong interactions with the helicene molecules. The $\mathrm{Ni}_{9} \mathrm{FeO}_{x}$ electrodes have much higher roughness compared to the $\mathrm{NiO}_{x}$ electrodes. The helicene molecules were probably inserted into the porous structures of the $\mathrm{Ni}_{9} \mathrm{FeO}_{x}$ electrodes. Nevertheless, the effect caused by the chiral molecules is independent and compatible with the Fe doping effect. 


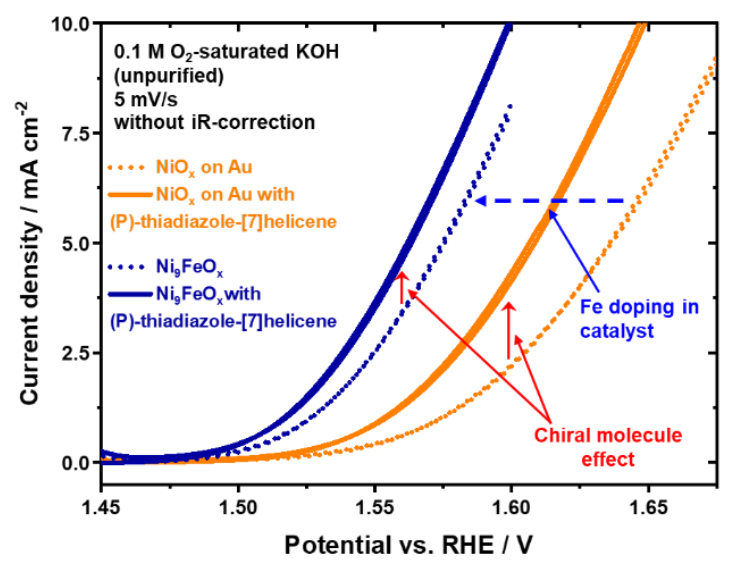

Fig. 2 | Comparison of chiral molecule effect with Fe-doping effect. Chiral molecule induced enhancement on $\mathrm{NiO}_{x}$ and $\mathrm{Ni}_{9} \mathrm{FeO}_{x}$.

To confirm the enhancement is owing to the chirality of the adsorbed helicene molecules instead of other neglected properties of thiadiazole-[7]helicene and, at the same time, to investigate the influence of the helicene length on the OER, we synthesized bis(thiadiazole)[8]helicene, which contains an additional thiadiazole ring at the opposite end of the helix, expected to stabilize the Au-helicene- $\mathrm{NiO}_{x}$ sandwich structure. We produced the racemic form of bis(thiadiazole)-[8]helicene and resolved the $(P)$ and $(M)$ enantiomers by chiral HPLC on Chiralpak IF (see Methods and the Supplementary Information for the synthesis, separation procedures and crystal structures). Suitable single crystals for the X-ray diffraction analysis have been obtained for both the enantiopure and also the racemic helicenes. The first eluted enantiomer was the dextrorotatory one, having a specific optical rotation of $[\alpha]_{\mathrm{D}}^{25}=+(8100 \pm 1 \%)^{\circ}$, corresponding, as expected, to the clockwise $(P)$ enantiomer, in agreement with the single crystal X-ray analysis. Mirror-image circular dichroism (CD) spectra were obtained for the two enantiomers (see Supplementary Figure 12). In the solid state, the helical curvature, characterizing the dihedral angle between the two terminal thiadiazole rings, amounts at $29^{\circ}$ for the racemate and $35^{\circ}$ for the enantiopure compounds, thus smaller than $45^{\circ}$ observed for the thiadiazole-[7]helicenes ${ }^{27}$. Then, with the 
new bis(thiadiazole)-[8]helicene in our hands, we set out to the chiral molecular functionalization of our electrodes.

Fig. 3 a shows that the activity of $\mathrm{NiO}_{x}$ on $\mathrm{Au}$ was improved by the presence of $(M)$ bis(thiadiazole)-[8]helicene molecules on the surface, e.g., ca. $131.5 \%$ at the potential of $1.65 \mathrm{~V}$ vs. RHE. The (M)-bis(thiadiazole)-[8]helicene yields a sensibly greater improvement in the OER current than the thiadiazole-[7]helicenes. The much more intense OER activity enhancement by the bis(thiadiazole)-[8]helicene functionalized electrodes compared to the thiadiazole-[7]helicene functionalized ones is in favor of this sandwich-type structure of the electrodes, with the helicene lying between the $\mathrm{Au}$ substrate and the $\mathrm{NiO}_{x}$ islands, since it can be hypothesized that the former strongly interacts with both the substrate and the catalyst thanks to the presence of the functional thiadiazole rings on both sides of the helical connector.

So far, the results show that chiral helicene molecules can enhance the OER activity. However, the compound in the molecules that directly bonds to the electrode surface is the thiadiazole group. Thus, it is essential to evaluate the role of thiadiazole. Accordingly, we used 2,1,3-benzothiadiazole, a simple achiral molecule containing a thiadiazole cycle fused to a benzene ring. It has the same molecular footprint bonds to the $\mathrm{Au}(111)$ surface as the helicene molecules used in this work. As shown in Fig. $\mathbf{3} \mathbf{b}$, the presence of the achiral molecules did not noticeably influence the OER activity of $\mathrm{NiO}_{x}$ islands on $\mathrm{Au}$. Therefore, the interaction between the thiadiazole compound and the electrode surface is not the origin of the OER enhancement activity. 

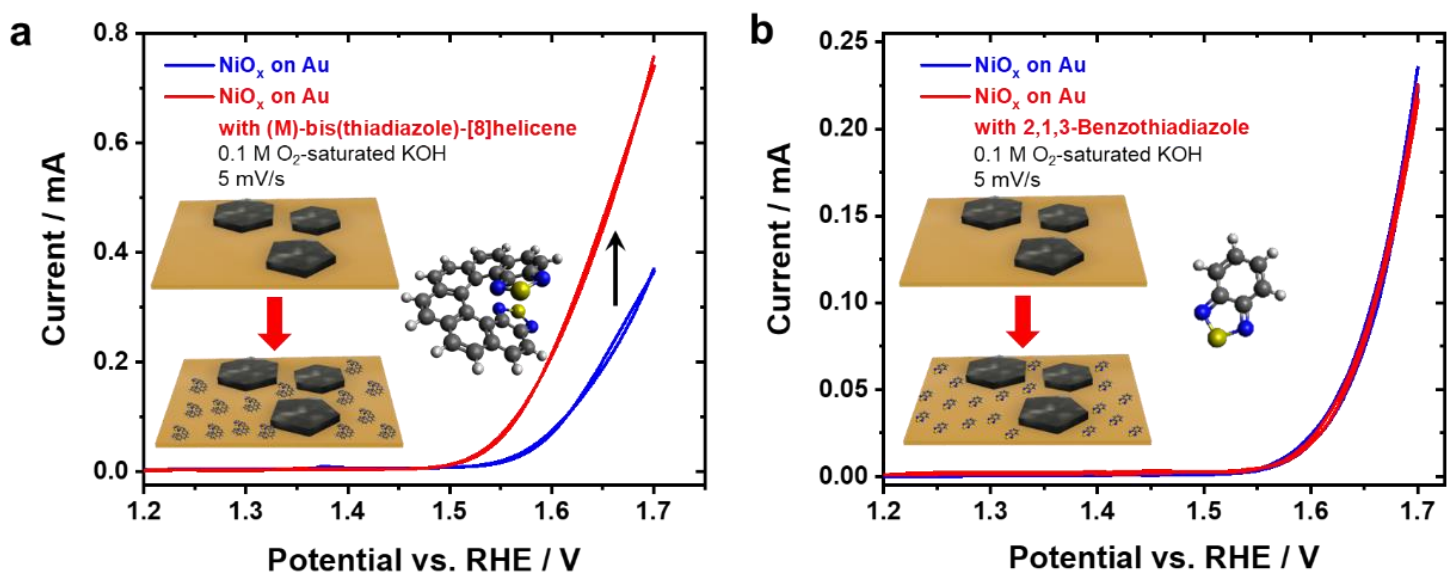

Fig. 3 | OER activity of $\mathrm{NiO}_{x}$ with chiral and achiral molecules. OER activity of a $(M)$ bis(thiadiazole)-[8]helicene on Au surface with $\mathrm{NiO}_{x}$, b 2,1,3-benzothiadiazole on Au with $\mathrm{NiO}_{\mathrm{x}}$ in $0.1 \mathrm{M} \mathrm{O}_{2}$-saturated $\mathrm{KOH}$.

On $\mathrm{Au}(111)$ surfaces, the helicene molecules formed self-assembled monolayer (SAM) structures. The STM images of $(P)$-thiadiazole-[7]helicene and $(M)$-bis(thiadiazole)[8]helicene molecules on $\mathrm{Au}(111)$ are shown in Fig. $\mathbf{4} \mathbf{a}$ and $\mathbf{b}$, respectively (see Supplementary Figure 3 for larger scale images showing different domains of assemblies). The former formed trimeric structures, while the latter formed rows of dimers. In the SAM of (M)-bis(thiadiazole)-[8]helicene molecules, the existence of three types of domains $\left(60^{\circ}\right.$ to each other) is observed, as shown in Supplementary Figure $\mathbf{3} \mathbf{b}$. These types of molecular assemblies are common to other functionalized helicenes on $\mathrm{Au}(111)^{21,22,23,25}$. The molecular coverage of functionalized helicenes on the substrates determines the assembly, going from trimeric structures at low coverage to dimeric rows at high coverage $22,23,24,25$. Therefore, the $(M)$-bis(thiadiazole)-[8]helicene reached a higher coverage than the $(P)$-thiadiazole[7]helicene under similar conditions, indicating that the (M)-bis(thiadiazole)-[8]helicene established stronger interactions with the Au substrate likely due to the added thiadiazole group. 

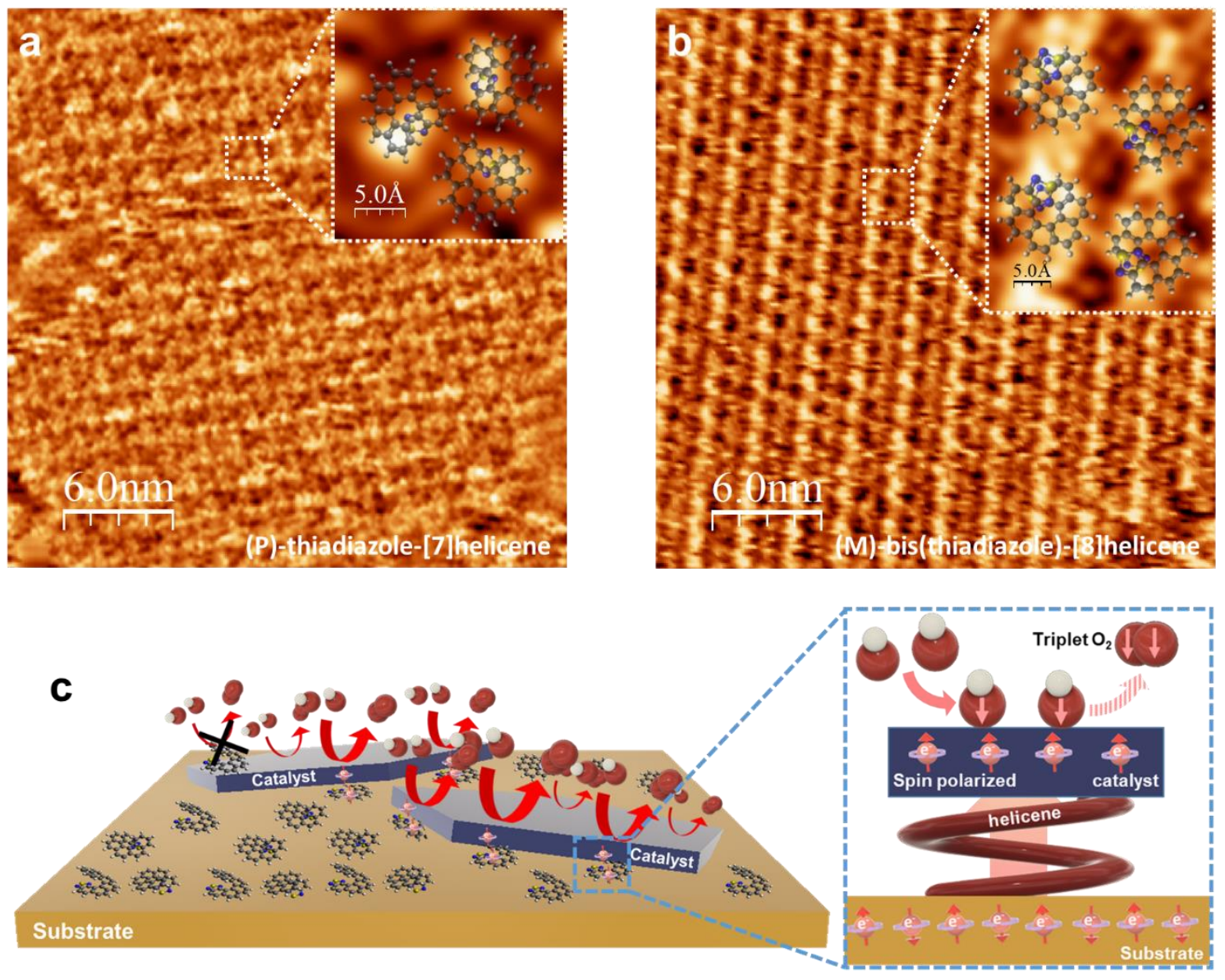

Fig. 4 | Helicene SAM and effect on the OER activity. a, an STM image of SAM of $(P)$ -

thiadiazole-[7]helicene (image size: $30 \mathrm{~nm} \times 30 \mathrm{~nm}$, tunneling current: $25 \mathrm{pA}$, sample bias: -5 $\mathrm{mV}$ ). The dashed square marks a trimer of the molecules. A high resolution image (image size: $2.2 \mathrm{~nm} \times 2.2 \mathrm{~nm}$, tunneling current: $20 \mathrm{pA}$, sample bias: $20 \mathrm{mV}$ ) showing one trimer is in the inset. $\mathbf{b}$, an STM image of SAM of $(M)$-bis(thiadiazole)-[8]helicene on Au(111) (image size: $30 \mathrm{~nm} \times 30 \mathrm{~nm}$, tunneling current: $20 \mathrm{pA}$, sample bias: $25 \mathrm{mV}$ ). The dashed rectangle marks two dimers of the molecules. The inset shows a high resolution image (image size: 3 $\mathrm{nm} \times 4 \mathrm{~nm}$, tunneling current: $20 \mathrm{pA}$, sample bias: $-25 \mathrm{mV}$ ) of two dimers. The 3D models of the molecules show the locations of the molecules. c Chiral molecule effect on the OER activity of $\mathrm{NiO}_{x}$ islands dependent on the catalyst- chiral molecule-Au substrate configuration and illustration of the chiral molecular spin polarization effect on the OER. 
STM images were taken on the helicene molecule functionalized $\mathrm{NiO}_{x}$ samples after the OER measurements, as shown in Supplementary Figure 4. The $\mathrm{NiO}_{x}$ islands were roughened due to the OER, and the surfaces of the islands were likely not covered by the molecules. However, the structures on the Au surface suggest that the helicene molecule assembly on Au was stable during the OER. Bare Au surfaces can be seen in several areas in the images, which is in line with the $\mathrm{Pb}$ stripping measurement results in Supplementary

\section{Figure 2.}

The activity enhancement using chiral molecules appears to depend on the anchoring of the molecules regarding to the catalysts. Based on our results, the dependence of the OER activity enhancement on the molecule-catalyst configuration is presented in Fig. $\mathbf{4}$ c. Helicene molecules on top of the catalyst surface block the active centers and consequently reduce the overall catalytic activity. Therefore, electrode manufacturing is vital to the performance of helicene molecule functionalized catalysts. A rationally designed electrode requires the catalytically active centers free of blockage and the electron transfer through the spin polarizers. For an optimal activity enhancement, the chiral molecules should be between the catalytic material and the substrate. A substrate that can strongly bond the chiral molecules (for example, Au in this work) is preferred for fabricating a stable electrode.

\section{Chiral molecular functionalization}

In this work, we show that chiral molecular functionalization can improve the OER activity of metal oxide catalysts. A specific catalyst-chiral helicene molecule-Au substrate configuration (as shown in Fig. 4c) shows the optimal catalytic activity. By comparing the effect of chiral helicene molecules and the achiral 2,1,3-benzothiadiazole, it is evident that such activity enhancement is directly related to the chirality of the molecules, rather than ligand effects introduced by the bonding of the molecules on the catalyst surface. The bonding between the catalyst and the chiral molecule alone does not cause any improvement in the catalytic 
activity. Contrarily, the bonding can reduce the activity when the chiral molecules are directly deposited at the catalyst active sites. The results of this comprehensive study are in agreement with the interpretation from Ref. 8 that a CISS-induced effect can efficiently enhance the OER.

Although often considered a spin filter, chiral molecules are better described as spin polarizers as the chirality does not reduce the transition probability. In electron scattering, the spin polarization is induced by symmetry breaking ${ }^{41}$. This can be expanded to any process that can be described by a transition matrix, for example, photoemission, where the spin polarization is induced by the broken experimental symmetry also for light elements ${ }^{42,43}$. In electron transport, all contributions from the complex transition matrix elements are typically symmetric unless a chiral element is introduced. In this case, the transmitted electrons will acquire a spin polarization, similar to a paramagnetic material in an external magnetic field. Garcés-Pineda et al. ${ }^{9}$ reported on the external magnetic field enhancement of the OER at various magnetic transition metal oxide-based catalysts. Although an activity enhancement can be caused by both chiral molecular functionalization and an external magnetic field, the enhancement magnitudes appear to be different. At the potential of 1.65 V vs. $\mathrm{RHE}$, the OER current density (scan rate: $5 \mathrm{mV} / \mathrm{s}$ ) on $\mathrm{NiO}_{x}$-based catalysts in $0.1 \mathrm{M}$ $\mathrm{KOH}$ increased ca. $61 \%$ by using $(P)$-thiadiazole-[7]helicene, which is significantly larger than the ca. $10 \%$ in $1 \mathrm{M} \mathrm{KOH}$ (scan rate: $5 \mathrm{mV} / \mathrm{s}$ ) caused by an external magnetic field ( $\leqslant$ $450 \mathrm{mT})^{9}$. Thus, chiral molecular functionalization may be relatively more effective. It has also been well established that external magnetic fields can accelerate mass transfer processes through a magnetohydrodynamic effect ${ }^{44,45,46}$. The local magnetic field at the catalyst surface introduced by the chiral molecules and its effect on the local mass transfer should be further probed.

It was suggested that the electron spin at the catalyst surface strongly influences the bonding strength of the catalyst to oxygen species and the charge transfer between the catalyst and the oxygen adsorbates ${ }^{9,47,48,49}$. Electron spin polarization at catalyst surfaces with specifically 
modulated ferromagnetic properties leads to advanced OER kinetics ${ }^{10,50,51,52}$, and a ferromagnetic catalyst can promote the spin polarization under an external magnetic field and thus enhances its OER activity ${ }^{53}$. However, not all practical OER catalysts are ferromagnetic. Additionally, metal oxide-based catalysts, especially highly active 2D nanostructured catalysts, undergo severe surface reconstruction processes under OER conditions ${ }^{54,55,56}$. The preservation of the well-prepared ferromagnetic properties under surface reconstruction is questionable. The chiral molecular functionalization provides a more versatile and sustainable electron spin polarization effect (Fig. 4 c). It can be applied to most catalysts independent of their electronic (magnetic) properties and under different reaction conditions, i.e., less affected by the catalyst composition and surface reconstruction.

Compared to chiral molecular functionalization, Fe doping is a verified way to improve the OER activity of Ni-based catalysts ${ }^{57}$. It is revealed that the OER activity of Ni-based catalysts in non-specifically treated electrolytes (containing a trace amount of $\mathrm{Fe}$ ) is affected by the $\mathrm{Fe}$ doping, and the catalysts effectively turn into NiFe dual catalysts ${ }^{37}$. Our results show that chiral molecular functionalization enhancement can coexist with the Fe-doping effect and other alloying methods since the helicene molecules do not interact directly with the metal oxide active centers. Therefore, it is compatible with most metal oxide-based catalysis systems and independent of the chemical composition of the catalysts.

Moreover, the newly introduced (M)-bis(thiadiazole)-[8]helicene brings a higher enhancement to the OER than (M)-thiadiazole-[7]helicene, i.e., ca. $131.5 \%$ vs. ca. $74 \%$ in the overall current at the potential of $1.65 \mathrm{~V}$ vs. $\mathrm{RHE}$ in $0.1 \mathrm{M} \mathrm{KOH}$. This finding suggests the enhancement is related to the structure of the helicene molecules, for instance, molecular length and functional groups that determine the interactions with the catalyst and the substrates. Further enhancement through modification of the helicene molecules can be anticipated. 


\section{Conclusions}

In summary, we have validated and evaluated the chiral molecular functionalization on the oxygen evolution at 2D hybrid chiral/achiral molecule-transition metal oxide electrodes. The results show that the chirality of the helicene molecules is accountable for a great enhancement in the activity of state-of-the-art OER catalysts. The enhancement is related to the electron spin polarization at the catalyst surface. However, the chiral molecular functionalization does not change the catalyst composition, and it is compatible with other enhancement methods, e.g., Fe doping. Therefore, this approach has the potential to boost electron spin-dependent catalytic reactions (e.g., the OER) beyond the common volcano-plot limits governed by the Sabatier principle and the scaling relationships. The comparison of different electrode configurations provides a clear guideline for optimizing the enhancement. This approach opens new avenues for developing next-generation catalytic systems with high efficiency and advanced selectivity through rational molecular functionalization.

\section{Methods}

Synthesis of (rac)- bis(thiadiazole)-[8]helicene. For the reaction Scheme, full experimental details, characterization, chiral HPLC separation and X-ray diffraction analysis details, see the Supplementary Information.

\section{2,7-bis(benzothiadiazol)vinyl)naphthalene 2.}

2,7-bis((triphenylphosphonium)methyl)naphthalene bromide 1 (0.26 g, $0.31 \mathrm{mmol}, 1$ equiv.) was suspended in $7 \mathrm{~mL}$ of dry THF under an argon atmosphere. The mixture was cooled down to $-78^{\circ} \mathrm{C}$ and then $n \mathrm{BuLi}(0.41 \mathrm{~mL}, 1.6 \mathrm{M}$ in hexane, $0.65 \mathrm{mmol}, 2.1$ equiv.) was added dropwise. After $30 \mathrm{~min}$ at $-78^{\circ} \mathrm{C}$, it was warmed to room temperature and stirred for one hour. The mixture was cooled down again to $-78^{\circ} \mathrm{C}$ and benzothiadiazole-5-carbaldehyde was added $\left(0.16 \mathrm{~g}, 0.097 \mathrm{mmol}, 3.1\right.$ equiv.). The mixture was stirred 10 minutes at $-78^{\circ} \mathrm{C}$, 
then warmed to room temperature and left at RT overnight. After filtration on Celite ${ }^{\circledR}$ and concentration under vacuum, column chromatography $\left(\mathrm{SiO}_{2}\right.$, petroleum ether: $\left.\mathrm{CH}_{2} \mathrm{Cl}_{2} 4: 6\right)$ yielded 2 as a yellow powder (90 mg, 45\% yield, mixture of Z/E isomers). ${ }^{1} \mathrm{H}$ NMR (300 $\left.\mathrm{MHz}, \mathrm{CDCl}_{3}\right): \delta(\mathrm{ppm})$ 9.07z (s, 0.1H), 8.20-7.60 (m, 10H), 7.56-7.33 (m, 4H), 7.20-6.80 (m, $2 \mathrm{H}) . \delta(\mathrm{ppm}) \mathrm{MS}(\mathrm{MALDI}) \mathrm{m} / \mathrm{z}=448.0$, theor. calc. $448.1\left(\mathrm{M}^{\bullet+}\right)$.

(rac)-bis(thiadiazole)-[8]helicene. 2,7-bis(benzothiadiazol)vinyl)naphthalene 2 (90 mg, 0.2 mmol, 1 equiv.) was dissolved in $350 \mathrm{~mL}$ of toluene in a photoreactor with a catalytic amount of iodine (ca. 0.1 equiv.) and the mixture was irradiated with an immersion lamp (150 W) for 20 hours. After evaporation of the solvent and column chromatography $\left(\mathrm{SiO}_{2}, \mathrm{PE}: \mathrm{CH}_{2} \mathrm{Cl}_{2}\right.$ 4:6) the compound was obtained as a yellow powder (40 mg, 44\%). ${ }^{1} \mathrm{H}$ NMR (300 MHz, $\left.\mathrm{CDCl}_{3}\right): \delta(\mathrm{ppm}) 8.17$ (d, J = 8.2 Hz, 2H), $8.08(\mathrm{~s}, 4 \mathrm{H}), 7.74(\mathrm{~d}, J=8.2 \mathrm{~Hz}, 2 \mathrm{H}), 7.51$ (d, J = $9.2 \mathrm{~Hz}, 2 \mathrm{H}), 7.38$ (d, J = 9.2 Hz, 2H). ${ }^{13} \mathrm{C}$ NMR (76 MHz, $\left.\mathrm{CDCl}_{3}\right) \delta(\mathrm{ppm})$ 153.76, 151.80, 133.25, 132.38, 132.18, 131.79, 129.81, 129.53, 128.54, 128.44, 127.84, 127.59, 126.17, 125.47, 123.66, 118.96. MS (MALDI) $\mathrm{m} / \mathrm{z}=444.0$, theor. calc. $444.0\left(\mathrm{M}^{\bullet+}\right)$.

Sample preparation. Monolayer $\mathrm{NiO}_{x}$ islands were synthesized by liquid exfoliation from the bulk counterparts reported elsewhere ${ }^{58,59}$ and spin-coated on $\mathrm{Au}(111)$ film on mica substrates (epitaxial gold on mica, Georg Albert PVD - Beschichtungen). $\mathrm{Ni}_{9} \mathrm{FeO}_{x}$ on $\mathrm{Ni}$ foil electrodes were synthesized using a combustion method ${ }^{40}$.

All molecules were first dissolved in dichloromethane (DCM, Sigma-Aldrich, puriss. p.a., $\geq 99.9 \%$ (GC)). The molecule solutions were drop-casted on the electrode surfaces to deposit a quasi-monolayer of molecules. After the DCM evaporated completely and all the molecules landed on the surfaces, the electrodes were rinsed with excessive DCM to remove molecules not adsorbed directly on the electrode surface. Afterwards, the electrodes 
were rinsed with Milli-Q water and ready for the OER activity measurement with adsorbed molecules.

The catalytic activity of Au surfaces is highly dependent on the surface morphology ${ }^{60}$. Under OER conditions, surface reconstruction continuously occurs on Au surfaces and strongly affects the OER activity ${ }^{36}$. Therefore, OER activity measurements of helicene moleculefunctionalized Au surface used freshly prepared Au substrates (Au(111) film on mica substrates). Au samples for bare Au experiments were treated using pure DCM following the helicene deposition procedure; however, without helicene molecules dissolved in DCM. This treatment is to rule out the effect of the deposition procedure on other surface properties (e.g., roughness) that may influence the activity comparison. Identical experiments (activity and $\mathrm{Pb}$ stripping measurements) were conducted on bare Au and helicene moleculefunctionalized Au.

Electrochemical characterization. The electrochemical measurements were conducted in a three-electrode cell. The overall activity depends on the absolute amount of $\mathrm{NiO}_{\mathrm{x}}$ deposited on the Au surface, which varies slightly between samples. An unpurified $\mathrm{KOH}$ solution ( $1 \mathrm{~N}$ solution in water, ACROS Organics ${ }^{\mathrm{TM}}$ ) has been used in this work. The measurements were kept running until stable CVs were observed. All electrochemical measurements were conducted using a VSP-300 (BioLogic) potentiostat. A coiled Au wire (99.9\%, Alfa Aesar) was used as the counter electrode (CE). The CE was cleaned by flame annealing and rinsed with Milli-Q (Millipore) water before each set of measurements. A HydroFlex® standard hydrogen reference electrode (Gaskatel) was used as the reference electrode (RE). The activity measurements were done without iR-correction to avoid overcompensation and only compare the effect of molecule deposition to the overall catalytic performance. 
STM measurements. STM has been used to measure the deposition of the helicene molecules. A Bruker MS10 EC-STM with a NanoScope ${ }^{\circ}$ V controller controlled by the software Nanoscope 8.15 has been used for the STM measurements. Image analysis was done using the WSxM v5.0 Develop 9.1 software $^{61}$ and molecular scale models using LMAPper and Avogadro software. Nonanoic acid was added on the sample surface as the solvent for a solid/liquid interface to obtain a greater resolution than the one achieved at the solid/air interface 62,63 .

\section{Data availability}

The data that support the findings of this study are available from the corresponding authors upon reasonable request. 


\section{References}

\footnotetext{
${ }^{1}$ Roger, I., Shipman, M. A. \& Symes, M. D. Earth-abundant catalysts for electrochemical and photoelectrochemical water splitting. Nat. Rev. Chem. 1, 0003 (2017).

2 Eisenberg, R., Gray, H. B. \& Crabtree, G. W. Addressing the challenge of carbon-free energy. Proc Natl Acad Sci USA 117, 12543-12549 (2020).

${ }^{3}$ Shiva Kumar, S. \& Himabindu, V. Hydrogen production by PEM water electrolysis - A review. Mater. Sci. Energy Technol. 2, 442-454 (2019).
}

${ }^{4}$ Che, M. Nobel Prize in chemistry 1912 to Sabatier: Organic chemistry or catalysis? Catal. Today 218, 162-171 (2013).

${ }^{5}$ Nørskov, J., Bligaard, T., Rossmeisl, J. et al. Towards the computational design of solid catalysts. Nat. Chem. 1, 37-46 (2009).

${ }^{6}$ Medford, A. J. et al. From the Sabatier principle to a predictive theory of transition-metal heterogeneous catalysis. J. Catal. 328, 36-42 (2015).

7 Pérez-Ramírez, J. \& López, N. Strategies to break linear scaling relationships. Nat Catal 2, 971-976 (2019).

${ }^{8}$ Zhang, W., Banerjee-Ghosh, K., Tassinari, F. \& Naaman, R. Enhanced Electrochemical Water Splitting with Chiral Molecule-Coated $\mathrm{Fe}_{3} \mathrm{O}_{4}$ Nanoparticles. ACS Energy Lett. 3, 2308-2313 (2018).

${ }^{9}$ Garcés-Pineda, F. A., Blasco-Ahicart, M., Nieto-Castro, D., López, N. \& Galán-Mascarós, J. R. Direct magnetic enhancement of electrocatalytic water oxidation in alkaline media. Nat Energy 4, $519-525$ (2019).

${ }^{10} \mathrm{Li}, \mathrm{X}$., Liu, H., Chen, Z. et al. Enhancing oxygen evolution efficiency of multiferroic oxides by spintronic and ferroelectric polarization regulation. Nat Commun 10, 1409 (2019).

${ }^{11}$ Naaman, R., Paltiel, Y. \& Waldeck, D. H. Chiral molecules and the electron spin. Nat Rev Chem 3, 250-260 (2019).

${ }^{12}$ Brandt, J., Salerno, F. \& Fuchter, M. The added value of small-molecule chirality in technological applications. Nat Rev Chem 1, 0045 (2017).

${ }^{13}$ Mtangi, W. et al. Control of Electrons' Spin Eliminates Hydrogen Peroxide Formation During Water Splitting. J. Am. Chem. Soc. 139, 2794-2798 (2017). 
${ }^{14}$ Chung, D. Y. et al. Dynamic stability of active sites in hydr(oxy)oxides for the oxygen evolution reaction. Nature Energy 5, 222-230 (2020).

${ }^{15}$ Love, J. C., Estroff, L. A., Kriebel, J. K., Nuzzo, R. G. \& Whitesides, G. M. Self-Assembled Monolayers of Thiolates on Metals as a Form of Nanotechnology. Chem. Rev. 105, 1103-1170 (2005).

${ }^{16}$ Grimm, O. C., Somaratne, R. M. D. S., Wang, Y., Kim, S. \& Whitten, J. E. Thiol adsorption on metal oxide nanoparticles. Phys. Chem. Chem. Phys. 23, 8309-8317 (2021).

17 Miner, E. M. \& Dincă, M. Metal-organic frameworks: Evolved oxygen evolution catalysts. Nat Energy 1, 16186 (2016).

${ }^{18}$ Song, F., Hu, X. Exfoliation of layered double hydroxides for enhanced oxygen evolution catalysis. Nat Commun 5, 4477 (2014).

${ }^{19}$ Ernst, K.-H. Stereochemical Recognition of Helicenes on Metal Surfaces. Acc. Chem. Res. 49, $1182-1190$ (2016).

${ }^{20}$ Barroso, J. et al. Revisiting the racemization mechanism of helicenes. Chem. Commun. 54, 188191 (2018).

${ }^{21}$ Balandina, T. et al. Self-assembly of an asymmetrically functionalized [6]helicene at liquid/solid interfaces. Chem. Commun. 49, 2207 (2013).

${ }^{22}$ Ascolani, H. et al. Van der Waals interactions in the self-assembly of 5-amino [6]helicene on $\mathrm{Cu}(100)$ and $\mathrm{Au}(111)$. Chem. Commun. 5013907-13909 (2014).

${ }^{23}$ van der Meijden, M. W. et al. Synthesis, Properties, and Two-Dimensional Adsorption Characteristics of [6]Hexahelicene-7-carboxylic acid. Chem. Eur. J. 22, 14633-14639 (2016). ${ }^{24}$ Fuhr, J. D. et al. Chiral expression of adsorbed (MP) 5-amino[6]helicenes: from random structures to dense racemic crystals by surface alloying. Chem. Commun. 53, 130-133 (2017).

${ }^{25}$ Seibel, J., Parschau, M. \& Ernst, K.-H. Two-Dimensional Crystallization of Enantiopure and Racemic Heptahelicene on Ag(111) and Au(111). J. Phys. Chem. C 118, 29135-29141 (2014). ${ }^{26}$ Krukowski, P. et al. Adsorption and Light Emission of a Racemic Mixture of [7]thiaheterohelicene2,13-carboxaldehyde on $\mathrm{Au}(111), \mathrm{Cu}(001)$, and $\mathrm{NiAl}(110)$ Surfaces Investigated Using a Scanning Tunneling Microscope. J. Phys. Chem. C 125, 9419-9427 (2021).

${ }^{27}$ Biet, T. et al. Triggering Emission with the Helical Turn in Thiadiazole-Helicenes. Chem. Eur. J. 23, 437-446 (2017). 
${ }^{28}$ Pop, F., Zigon, N. \& Avarvari, N. Main-Group-Based Electro- and Photoactive Chiral Materials.

Chem. Rev. 119, 8435-8478 (2019).

${ }^{29}$ Farhat, R., Dhainy, J. \& Halaoui, L. I. OER Catalysis at Activated and Codeposited NiFe-

Oxo/Hydroxide Thin Films Is Due to Postdeposition Surface-Fe and Is Not Sustainable without Fe in Solution. ACS Catal. 10, 20-35 (2020).

30 Watzele, S. et al. Determination of Electroactive Surface Area of Ni-, Co-, Fe-, and Ir-Based Oxide Electrocatalysts. ACS Catal. 9, 9222-9230 (2019).

31 Tounsi, M. et al. Electrochemical Capacitive K+ EMIS Chemical Sensor Based on the Dibromoaza[7]helicene as an lonophore for Potassium Ions Detection. Electroanalysis 28, 2892-2899 (2016).

32 Tounsi, M. et al. A novel EIS field effect structures coated with TESUD-PPy-PVCdibromoaza[7]helicene matrix for potassium ions detection. Mater. Sci. Eng. C 61, 608-615 (2016). ${ }^{33}$ Sadler, J. E. et al. Surface functionalization of electro-deposited nickel. Phys. Chem. Chem. Phys. 13, 17987 (2011).

${ }^{34}$ Mitchell, C., Fayette, M. \& Dimitrov, N. Homo- and hetero-epitaxial deposition of Au by surface limited redox replacement of $\mathrm{Pb}$ underpotentially deposited layer in one-cell configuration. Electrochim. Acta 85, 450-458 (2012).

35 Jeyabharathi, C., Zander, M. \& Scholz, F. Underpotential deposition of lead on quasi-spherical and faceted gold nanoparticles. J. Electroanal. Chem. 819, 159-162 (2018).

${ }^{36}$ Zwaschka, G. et al. Imaging the Heterogeneity of the Oxygen Evolution Reaction on Gold

Electrodes Operando: Activity is Highly Local. ACS Catal. 10, 6084-6093 (2020).

${ }^{37}$ Trotochaud, L., Young, S. L., Ranney, J. K. \& Boettcher, S. W. Nickel-Iron Oxyhydroxide OxygenEvolution Electrocatalysts: The Role of Intentional and Incidental Iron Incorporation. J. Am. Chem. Soc. 136, 6744-6753 (2014).

${ }^{38}$ Dionigi, F. \& Strasser, P. NiFe-Based (Oxy)hydroxide Catalysts for Oxygen Evolution Reaction in Non-Acidic Electrolytes. Adv. Energy Mater. 6, 1600621 (2016).

${ }^{39}$ Song, F. et al. An Unconventional Iron Nickel Catalyst for the Oxygen Evolution Reaction. ACS Cent. Sci. 5, 558-568 (2019).

${ }^{40}$ Garces-Pineda, F. A. et al. Push-pull electronic effects in Surface-active sites enhance electrocatalytic oxygen evolution on transition metal oxides. ChemSusChem 14, 1595-1601 (2021)

${ }^{41}$ Kessler, J. Polarized Electrons (Springer, 1985). 
${ }^{42}$ Heinzmann, U. \& Dil, J. H. Spin-orbit-induced photoelectron spin polarization in angle-resolved photoemission from both atomic and condensed matter targets. J. Phys. Condens. Matter 24, 173001 (2012).

${ }^{43}$ M. Fanciulli et al., Spin Polarization and Attosecond Time Delay in Photoemission from Spin Degenerate States of Solids. Phys. Rev. Lett. 118, 067402 (2017).

${ }^{44} \mathrm{Li}$, Y., Zhang, L., Peng, J., Zhang, W. \& Peng, K. Magnetic field enhancing electrocatalysis of $\mathrm{Co}_{3} \mathrm{O}_{4} / \mathrm{NF}$ for oxygen evolution reaction. J. Power Sources 433, 226704 (2019).

${ }^{45}$ L. M. A. Monzon, J. M. D. Coey, Magnetic fields in electrochemistry: The Lorentz force. A minireview. Electrochem. Commun. 42, 38-41 (2014).

${ }^{46}$ V. Gatard, J. Deseure, M. Chatenet. Use of magnetic fields in electrochemistry: a selected review. Current Opinion Electrochem. 23, 96-105 (2020).

${ }^{47}$ Suntivich, J., May, K. J., Gasteiger, H. A., Goodenough, J. B. \& Shao-Horn, Y. A perovskite oxide optimized for oxygen evolution catalysis from molecular orbital principles. Science 334, 1383-1385 (2011).

${ }^{48}$ Gracia, J. Spin dependent interactions catalyse the oxygen electrochemistry. Phys. Chem. Chem. Phys. 19, 20451-20456 (2017).

${ }^{49}$ Gracia, J. Itinerant spins and bond lengths in oxide electrocatalysts for oxygen evolution and reduction reactions. J. Phys. Chem. C 123, 9967-9972 (2019).

${ }^{50}$ Chen, R. R. et al. Antiferromagnetic inverse spinel oxide $\mathrm{LiCoVO}_{4}$ with spin-polarized channels for water oxidation. Adv. Mater. 32, 1907976 (2020).

${ }^{51}$ Sun, Y. et al. Spin-related electron transfer and orbital interactions in oxygen electrocatalysis. Adv. Mater. 32, 2003297 (2020).

${ }^{52} \mathrm{Wu}, \mathrm{T}$., Ren, X., Sun, Y. et al. Spin pinning effect to reconstructed oxyhydroxide layer on ferromagnetic oxides for enhanced water oxidation. Nat Commun 12, 3634 (2021).

${ }^{53}$ Ren, X., Wu, T., Sun, Y. et al. Spin-polarized oxygen evolution reaction under magnetic field. Nat Commun 12, 2608 (2021).

${ }^{54}$ Deng, J. et al. Morphology dynamics of single-layered $\mathrm{Ni}(\mathrm{OH})_{2} / \mathrm{NiOOH}$ nanosheets and subsequent Fe incorporation studied by in situ electrochemical atomic force microscopy. Nano Lett. 17, 69226926 (2017). 
${ }^{55}$ Dette, C., Hurst, M. R., Deng, J., Nellist, M. R. \& Boettcher, S. W. Structural evolution of metal (oxy)hydroxide nanosheets during the oxygen evolution reaction. ACS Appl. Mater. Interfaces 11, 5590-5594 (2019).

${ }^{56}$ Gao, L., Cui, X., Sewell, C. D., Li, J. \& Lin, Z, Recent advances in activating surface reconstruction for the high-efficiency oxygen evolution reaction. Chem. Soc. Rev. 50, 8428-8469 (2021).

${ }^{57}$ Anantharaj, S., Kundu, S. \& Noda, S. "The Fe Effect": A review unveiling the critical roles of Fe in enhancing OER activity of $\mathrm{Ni}$ and Co based catalysts. Nano Energy 80, 105514 (2021).

58 Ida, S., Shiga, D., Koinuma, M. \& Matasumto, Y. Synthesis of hexagonal nickel hydroxide nanosheets by exfoliation of layered nickel hydroxide intercalated with dodecyl sulfate ions. J. Am. Chem. Soc. 130, 14038-14039 (2008).

${ }^{59}$ Song, F. \& Hu, X. Exfoliation of layered double hydroxides for enhanced oxygen evolution catalysis. Nat Commun 5, 4477 (2014).

${ }^{60}$ Liu, L. \& Corma, A. Metal catalysts for heterogeneous catalysis: from single atoms to nanoclusters and nanoparticles. Chem. Rev. 118, 4981-5079 (2018).

${ }^{61}$ Horcas, I. et al. WSXM: A software for scanning probe microscopy and a tool for nanotechnology. Rev. Sci. Instrum. 78, 013705 (2007).

${ }^{62}$ Cometto, F. et al. The STM bias voltage-dependent polymorphism of a binary supramolecular network. Chem. Commun. 53, 11430-11432 (2017).

${ }^{63}$ Ubink, J., Enache, M. \& Stöhr, M. Bias-induced conformational switching of supramolecular networks of trimesic acid at the solid-liquid interface. J. Chem. Phys. 148, 174703 (2018). 


\section{Acknowledgements}

We heartily thank Prof. Hugo Dil for sharing his input on the discussion of the CISS effect in terms of spin polarization. S.L. acknowledges financial support by the Marie Skłodowska-Curie Fellowship (No. 838367 to S.L.) under the European Union's Horizon 2020 research. K.M., N.Z., N.V. and N.A. acknowledge the support from the CNRS, the University of Angers and the RFI LUMOMAT (grant to K.M.) in France. J.R.G.M. and F.A.G.P. thank the financial support of the FEDER/Ministerio de Ciencia e Innovación, Agencia Estatal de Investigación (RTI2018-095618-B-I00); the Generalitat de Catalunya (2017-SGR-1406), the CERCA Programme/Generalitat de Catalunya.

\section{Author contributions}

Y.L., K.B., N.A, and M.L. conceived and designed the experiments. N.A. and M.L. supervised the project and led the collaboration efforts. Y.L., K.B., K.M., N.Z., and N.V. carried out the experiments and analyzed the data. S.L., F.A.G.P., J.R.G.M., synthesized the catalysts. All authors participated in the result discussions. The manuscript was written by Y.L., N.A., and M.L. with contributions from all the authors. 